\section{Estudo longitudinal do nível de atividade física de mulheres idosas}

\section{Longitudinal study of physical activity level of older adult women}

Gisele Antunes do Livramento

Pallomma Patrícia Andressa Nart Fagundes ${ }^{1}$

Gabriele Regiane Winter ${ }^{2}$

Vanessa Porto Bernardes ${ }^{1}$

Maressa Priscila Krause ${ }^{2}$

\section{Resumo}

O objetivo deste estudo foi analisar as alterações longitudinais no nível de atividade física (NAF) de mulheres idosas. Este estudo longitudinal conduziu a primeira avaliação em 2005 e 2006, e a segunda avaliação em 2011, na qual participaram 78 mulheres (73,2 $\pm 5,2$ anos). O NAF foi avaliado através do Modified Baecke Questionnaire for Older Adults, o qual inclui os domínios: doméstico, esportivo e recreativo. A descrição da amostra foi realizada pela média e desvio padrão. A análise dos dados foi realizada por uma MANOVA e do teste multivariado de Hotelling's trace afim de verificar o efeito do tempo sobre os domínios do NAF ( $p<0,05)$. O teste multivariado de Hotelling's trace indicou diferenças significativas da variável independente (efeito do tempo: avaliações 1 e 2) $\left(\mathrm{F}_{1,154}=2,923 ; \mathrm{p}=0,036\right)$, para o NAF total $\left(\mathrm{F}_{1,154}=5,449 ; \mathrm{p}=0,021\right)$, e domínios doméstico $\left(\mathrm{F}_{1,154}=4,325 ; \mathrm{p}=0,039\right)$ e recreativo $\left(\mathrm{F}_{1,154}=5,299 ; \mathrm{p}=0,023\right)$. O NAF total alterou em -16,7\%, NAF doméstico em -7,6\%, NAF recreativo em -48,6\% (todos com p $<0,05$ ); e o NAF esportivo declinou $12,0 \%\left(\mathrm{~F}_{1,154}=0,892 ; \mathrm{p}=0,346\right)$. Concomitantemente ao avanço da idade, ocorre um declínio nos domínios do NAF de idosas, seja em atividades relacionadas ao cuidado da residência, exercícios físicos, ou atividades recreativas. Esses resultados demonstram a necessidade de maximizar politicas públicas para o cuidado à saúde do idoso a fim de promover atividades físicas nos diferentes domínios, e motivar idosos a manterem uma vida ativa e independente, principalmente participando regularmente de um programa de exercícios físicos, beneficiando a qualidade e os anos adicionais de vida e desta população.

\section{Palavras-chave}

Envelhecimento, Atividade física, Estudo longitudinal.

\begin{abstract}
The aim of this study was to analyze longitudinal changes on physical activity level (PAL) of older adult women. This longitudinal study conducted the first evaluation on 2005 and 2006, and the second evaluation on 2011, in which 78 older women (73.2 \pm 5.2 yrs) participated in the follow-up evaluation. PAL was evaluated by the Modified Baecke Questionnaire for Older Adults, which includes the domestic, sportive and recreational domains. Data was described by mean and standard deviation, and was analyzed by using a MANOVA and the Hotelling's trace multivariate test with the purpose to verify a time main effect on PAL domains ( $p<0.05)$. The multivariate test indicated significant differences for the independent variables (time effect: baseline and follow-up evaluations; $F_{1,154}=2,923 ; p=0.036$ ) for PAL-total $\left(F_{1,154}=5,449 ; p=0.021\right)$, PAL-domestic $\left(F_{1,154}=4,325 ; p=0.039\right)$ and PAL-leisure $\left(F_{1,154}=5,299 ; p=0.023\right)$. The PAL-total changed in $-16.7 \%$, PAL-domestic in -7.6\%, PAL-leisure in $-48.6 \%$ (all $p<0.05)$; and the PAL-sportive declined in $12.0 \%\left(F_{1,154}=0.892 ; p=0.346\right)$. Concurrently with aging, there is a declined on PAL domains that could be related to domestic, exercise, or recreational activities. These results demonstrated the need to maximize public health policies with the purpose to improve the health status of older adults by increasing the PAL on different domains, and also to motivate older adults to maintain an active and independent lifestyle, mainly enrolling in exercise program, leading to positive effects on quality of life, and on the additional years of life of this population.
\end{abstract}

\section{Keywords}

Aging, Physical activity, Longitudinal study.
Rev Bras Ativ Fis Saúde p. 552-561 DOI: http://dx.doi.org/10.12820/23171634.2012v17n6p552

1 Universidade Federal do Paraná; Curitiba, $P R$, Brasil

2 Grupo de Pesquisa em Fisiologia da Atividade Física e Saúde, Universidade Tecnológica Federal do Paraná; Curitiba, $P R$ Brasil.
Você pode ter acesso a este artigo na sua versão em inglês no site da Sociedade Brasileira de Atividade Física \& Saúde (www.sbafs.org.br) 


\section{INTRODUÇÃO}

O processo de envelhecimento acarreta mudanças físicas e morfológicas que podem estar associadas a incapacidades, independentemente ou não de doenças crônico degenerativas em idosos ${ }^{1}$. Dentre os fatores de melhoria, manutenção ou atenuação da redução da capacidade funcional e aptidão física, a prática regular de exercícios físicos é vista como um dos principais fatores para o sucesso deste processo $^{2}$. Apesar dos benefícios evidentes de um estilo de vida ativo, pesquisas demonstram uma redução no nível de atividade física entre idosos em inúmeros países desenvolvidos e em desenvolvimento ${ }^{3,4,5}$.

Segundo Eyler et al (2003) $)^{5}$, menos de $25 \%$ dos idosos, com 65 anos ou mais de idade, acumulam o mínimo recomendado de exercícios físicos nos Estados Unidos. Dados Brasileiros reportados pelo Ministério da Saúde 6 , demonstram que cerca de 60\% de adultos entre 39-69 anos mantêm hábitos insuficientes de atividade física. Segundo este levantamento, a inatividade física poderá ocasionar em 2020, 73\% de mortes atribuídas às doenças crônicas. Por sua vez, a publicação mais recente do Sistema de Vigilância de Fatores de Risco e Proteção para Doenças Crônicas

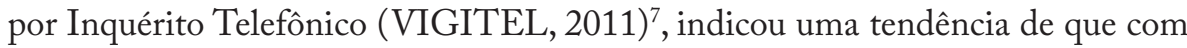
o avanço da idade, as mulheres diminuam as atividades realizadas no tempo livre ou de lazer. Apenas 9,4\% das mulheres idosas, especificamente na faixa etária de 65 anos ou mais de idade, foram consideradas ativas.

Baseando-se nos dados apresentados, verifica-se que concomitantemente ao avanço da idade, as mulheres diminuem seus níveis de atividade física. ${ }^{8}$. Contudo, Krause et al $(2007)^{9}$ reportaram dados que discordam parcialmente com esta tendência, ao comparar as alterações no NAF em mulheres com idade superior a 60 anos, as quais foram divididas em grupos de faixas etárias com intervalo de cinco anos. Observou-se que entre a faixa etária mais jovem para a mais idosa, ocorreu uma diminuição na realização de atividades físicas mais vigorosas, relacionada à prática de exercícios físicos; e um aumento de atividades com baixa demanda energética, relacionada a atividades recreativas. Segundo os autores, tais resultados poderiam indicar que com o avanço da idade, as mulheres idosas tenderiam a transitar de atividades que requerem mais esforço físico para aquelas de menor esforço, ou seja, idosas diminuem o nível de atividade física de intensidade moderada, e principalmente vigorosa.

As inconsistências dos resultados apresentados pode ser explicada pela escassez dos estudos nacionais que analisaram tais alterações, principalmente a partir de dados longitudinais, bem como a magnitude desta alteração nos diferentes domínios do NAF. Até o momento foi verificado que a maior proporção de atividade física insuficiente, ou sedentarismo, é encontrada em mulheres idosas e de baixo nível socioeconômico ${ }^{10}$; porém, escassos são os estudos brasileiros que investigaram tais fatores ${ }^{11,12,13,14,15,16}$. Sendo assim, o objetivo deste estudo foi analisar as alterações longitudinais em diferentes domínios de atividade física de mulheres idosas.

\section{MÉTODOS}

\section{Desenho do Estudo}

O delineamento da pesquisa é caracterizado como Estudo Longitudinal, com duas avaliações: a primeira avaliação foi iniciada em 2005 e finalizada em 2006, e a segunda avaliação, foi realizada no primeiro semestre de 2011 - intervalo médio de 5,8 anos. 


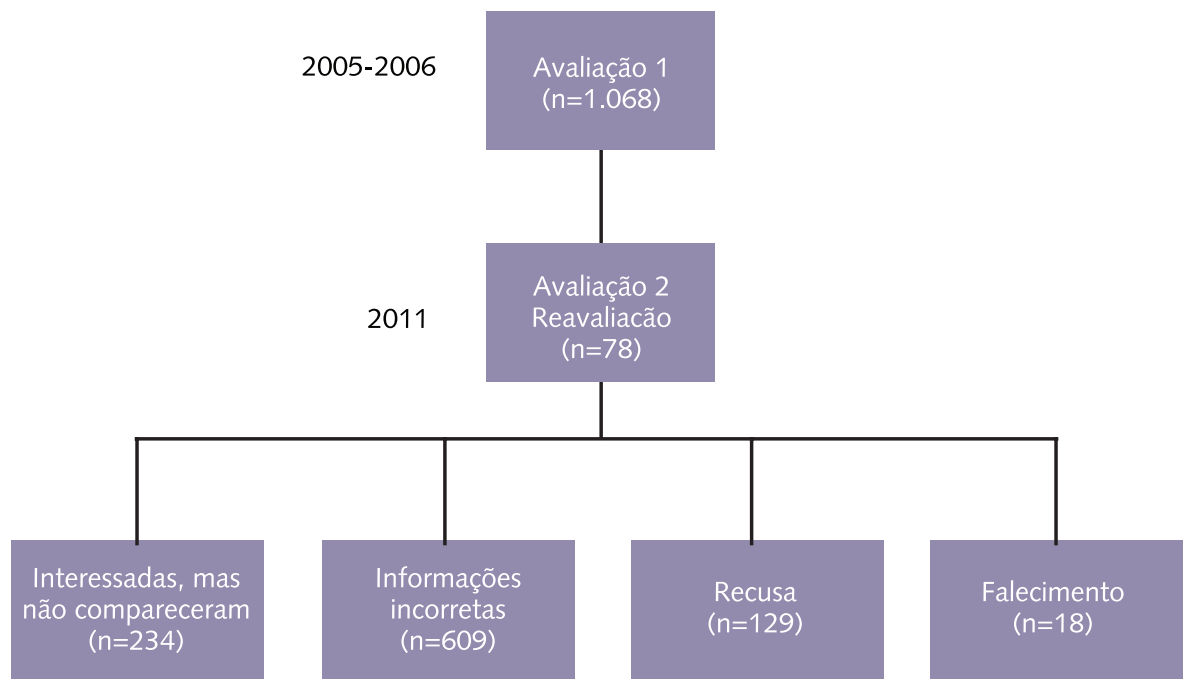

Figura 1 - Fluxograma das avaliações.

\section{População e Amostra}

O presente estudo foi realizado na cidade de Curitiba - Paraná, sendo parte integrante do Projeto Terceira Idade Independente (Centro de Pesquisa em Fisiologia da Atividade Física e Saúde - Universidade Tecnológica Federal do Paraná). A população do estudo foi constituída pelos participantes da primeira avaliação ( $\mathrm{n}=1068)$, os quais deveriam atender os seguintes critérios de inclusão: sexo feminino, idade superior a 60 anos, e viverem na comunidade (não-institucionalizada). Nenhum participante foi excluído deste estudo. Explicações detalhadas sobre o processo para seleção amostral foi publicado previamente por Krause et al (2007) ${ }^{9}$. O procedimento inicial da segunda avaliação (reavaliação) objetivou o contato indireto com os potencias participantes (1.068) via telefone. Além disso, a equipe de pesquisadores buscou o contato direto dos participantes que não foram contatados via telefone, deslocando-se até o endereço residencial. Mesmo com tais procedimentos, ocorreu uma perda amostral de mais da metade dos sujeitos devido a desatualização das informações pessoais. Durante o contato inicial, um membro treinado do grupo de investigadores informou os propósitos do estudo, possíveis benefícios e riscos atrelados e, após esclarecimento de todas as dúvidas, a reavaliação foi agendada. No momento da reavaliação, um investigador repetiu todas as informações relacionadas a pesquisa e esclareceu qualquer dúvida ainda existente, então, solicitou ao participante que assinasse o termo de consentimento livre e esclarecido, condicionando sua participação de modo voluntário. A amostra final da reavaliação foi constituída por 78 participantes (atualmente com idade superior a 65 anos).

O protocolo da segunda avaliação desta pesquisa foi submetido e aprovado ao Comitê de Ética da Pontifícia Universidade Católica do Paraná (CEP No 0004798/11), conforme as normas estabelecidas na Declaração de Helsinki e na Resolução 196/96 do Conselho Nacional de Saúde sobre pesquisa envolvendo seres humanos.

\section{Procedimentos}

Em ambas as avaliações, todos os procedimentos foram igualmente conduzidos. Com o intuito de evitar à influência de variações circadianas todas as avaliações foram realizadas em um mesmo período do dia (entre $08 \mathrm{~h} 00$ e 10h00). Os participantes foram instruídos a não realizarem atividade física vigorosa no dia anterior, como também a não ingerirem alimentos por um período de duas horas 
antecedentes ao seu início. Todas as avaliações foram realizadas no Departamento Acadêmico de Educação Física, da Universidade Tecnológica Federal do Paraná.

\section{Nível Socioeconômico - NSE}

O nível socioeconômico foi definido pelo critério de classificação econômica Brasil (Associação Nacional das Empresas de Pesquisa - ANEP, 2003) ${ }^{17}$. O resultado classifica o nível socioeconômico em sete classes $^{17}$ : E (0-5 pontos), D (6-10 pontos), C (11-16 pontos), B2 (17-20 pontos), B1 (21-24 pontos), A2 (25-29 pontos) e A1 (30-34 pontos). Posteriormente, três categorias foram compostas: Alta (A1 e A2), Média (B1 e B2) e Baixa (C, D e E).

\section{Variáveis Antropométricas}

As variáveis antropométricas foram obtidas conforme procedimentos propostos por Lohman et $\mathrm{al}^{18}$. Para a determinação da estatura (em metros $-\mathrm{m}$ ), o indivíduo avaliado permaneceu em posição ortostática com os pés unidos, descalço, trajando roupas esportivas leves (shorts e camiseta ou top). Além disso, o avaliado deveria manter-se em apneia inspiratória e com a cabeça orientada em $90^{\circ}$ conforme o plano de Frankfurt, tendo as superfícies do calcanhar, cintura pélvica, cintura escapular e região occipital em contato com o instrumento de medida, o qual encontrava-se fixado à parede. A massa corporal (MC, em quilogramas) foi mensurada com o avaliado permanecendo em posição ortostática, descalço, e trajando roupas esportivas leves (shorts e camiseta ou top). A massa corporal deveria permanecer distribuída entre os membros inferiores durante a permanência na plataforma do instrumento de medida (balança mecânica com estadiômetro, marca Welmy, modelo 104A; precisão de 0,1 quilogramas).

\section{Variável Dependente - Nível de Atividade Física (NAF)}

As informações foram obtidas após administração do Modified Baecke Questionnaire for Older Adults, adaptado para sujeitos idosos, conforme padronização de Voorrips et $\mathrm{a}^{19}$. Apesar das evidentes limitações devido à subjetividade empregada nas respostas do questionário, este instrumento tem apresentado uma correlação com outros métodos de quantificação de atividade física ${ }^{20}$, como o recordatório de atividades em 24 horas $(\mathrm{r}=0,78)$ e pedômetro $(\mathrm{r}=0,72)$. Além disso, o Modified Baecke Questionnaire for Older Adults foi validado especificamente para idosos brasileiros, apresentando bons indicadores de reprodutibilidade $(\mathrm{r}=0,89)^{21,22}$.

O questionário foi aplicado em formato de entrevista, sendo composto por 12 itens envolvendo atividades físicas domiciliares cotidianas (NAF_D), recreativas (NAF_R) e esportivas (NAF_E). Todos os avaliadores foram previamente treinados com o intuito de diminuir a variabilidade inter-avaliadores. $\mathrm{O}$ domínio doméstico é avaliado através de perguntas com opções objetivas de resposta, abrangendo atividades de 1) limpeza da casa de intensidade leve, como "tirar o pó da mobília", à intensidades vigorosas, como "esfregar o chão para limpar pisos"; 2) transporte, o qual indica a forma de se deslocar para fazer compras, seja caminhando, utilizando uma bicicleta, carro ou transporte público; 3) subir e descer escadas; e, 4) preparo de refeições. Os domínios esportivo e recreativo necessitam que o avaliado informe o tipo de atividade (nome), intensidade, frequência (horas/ semana) e tempo de prática (meses/ano). Posteriormente, o escore de cada domínio é calculado baseado na demanda energética da atividade (em METS) - quanto menor o gasto energético, menor será a pontuação ${ }^{19}$. 


\section{Análise Estatística}

Todas as análises foram conduzidas utilizando o Statistical Package for the Social Sciences (SPSS, 18.0) for Windows. A distribuição dos dados foi verificada com o teste Kolmogorov-Smirnov, indicando a normalidade para o NAF_D na primeira avaliação, NAF_T e NAF_D na segunda avaliação. Apesar das demais variáveis não apresentarem distribuição normal, optou-se por realizar os testes paramétricos devido a característica da escala de medida das mesmas. Portanto, a descrição dos dados é apresentada através da média e desvio-padrão. A análise dos dados foi realizada através de uma $M A N O V A$ com medidas repetidas e do teste multivariado de Hotelling's trace a fim de verificar o efeito do tempo sobre os domínios do NAF. Para todas as análises foi adotado o nível de significância $p<0,05$.

\section{RESULTADOS}

A variação temporal média entre as avaliações do estudo foi de 5,8 anos. O nível socioeconômico demonstrou-se um pouco mais elevado na segunda avaliação; contudo, ambos os resultados classificam os participantes na classe social $\mathrm{C}$ ou baixa. A massa e estatura corporal apresentaram-se estáveis.

O teste multivariado de Hotelling's trace indicou diferenças significativas da variável independente (tempo: avaliação 1 e 2) $(\mathrm{p}=0,036)$ sobre as seguintes variáveis dependentes: o nível de atividade física total (NAF_T), resultante da soma dos escores obtidos em todos os domínios, alterou em $-16,7 \%\left(\mathrm{~F}_{1,154}=5,449 ; \mathrm{p}=0,021\right)$. Todos os domínios do NAF declinaram; sendo significativos apenas no NAF_D $\left(2,35 \pm 0,57\right.$ para $2,17 \pm 0,48$ pontos; $\left.F_{1,154}=4,325 ; p=0,039\right)$ e no NAF_R $(1,09 \pm 1,66$ para $0,56 \pm 1,16$ pontos; $\left.F_{1,154}=5,299 ; p=0,023\right)$. Contudo, o NAF_E, que representa atividades classificadas como exercícios físicos praticados regularmente, alterou de $2,83 \pm 2,20$ pontos para $2,49 \pm 2,26$ pontos $\left(\mathrm{F}_{1,154}=0,892 ; \mathrm{p}=0,346\right)$.

Tabela 1 - Características descritivas dos participantes - média (desvio-padrão).

\begin{tabular}{lcc}
\hline & Avaliação 1 & Avaliação 2 \\
\hline Idade (anos) & $67,2(5,4)$ & $73,2(5,2)$ \\
\hline NSE (pontos) & $13,8(4,5)$ & $14,2(4,0)$ \\
\hline Massa Corporal $(\mathrm{kg})$ & $68,5(11,0)$ & $68,4(12,1)$ \\
\hline \multicolumn{1}{c}{ Estatura Corporal $(\mathrm{m})$} & $1,54(0,06)$ & $1,54(0,06)$ \\
\hline
\end{tabular}

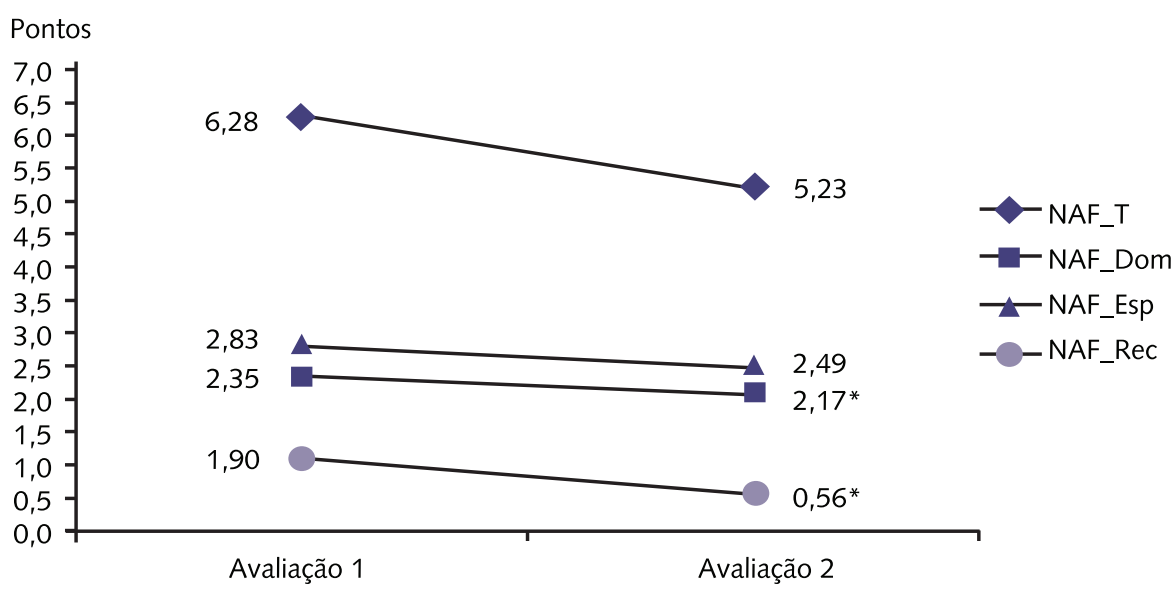

Figura 2 - Alterações longitudinais nos domínios do nível de atividade física. *Diferença significativa entre as avaliações $(p<0,05)$. 


\section{DISCUSSÃO}

As atividades comumente realizadas pela maioria dos idosos estão relacionadas aos domínios doméstico (NAF_D), esportivo (NAF_E) e recreativo (NAF_R ${ }^{23}$. Os domínios esportivo e recreativo compreendem atividades realizadas no tempo livre, relacionadas à prática regular de exercício físico e atividades de lazer/recreação, respectivamente. O domínio que demonstrou maior alteração foi o recreativo $(-48,6 \%, p<0,05)$. Um exemplo do escore obtido pelas participantes é a realização de uma atividade manual na posição sentada, de intensidade leve, 2 horas semanais, 9 meses de prática ( 0,68 pontos). As principais atividades reportadas foram: bingo, leitura, dança de salão em bailes e trabalhos manuais como costura, artesanato e pintura. Em geral, essas atividades são realizadas em um ambiente externo à residência, as quais implicam em uma exposição social, podendo favorecer a interação com outros indivíduos da mesma faixa etária que possuem interesses similares. Tais interações favorecem no desenvolvimento de novas amizades, de identidade com o grupo em que está inserido, promovendo a convivência e, consequentemente, beneficiando aspectos psicossociais ${ }^{24,25,26}$. Sendo assim, a grande magnitude de alteração de tais atividades é preocupante, pois indica que idosas podem estar se excluindo socialmente, minimizando a relação com outros indivíduos.

Por sua vez, o NAF_D apresentou o menor declínio dos domínios estudados $(-7,6 \% ; \mathrm{p}<0,05)$. Um escore similar ao obtido (de 2,0 pontos) corresponde a um indivíduo que realize frequentemente as atividades domésticas podendo ser auxiliado por outra pessoa. Tais resultados corroboram com as características socioculturais da sociedade Brasileira, na qual a atual geração de idosas teria a responsabilidade do cuidado do ambiente domiciliar ${ }^{16,27}$. Portanto, a redução ou a desistência em executar tais atividades podem ser consideradas essenciais à vida diária de idosas, comprometendo a autonomia e qualidade de vida das mesmas ${ }^{19,27,28}$. Houve também um declínio das atividades relacionadas a prática regular de exercício físico (NAF_E: -12\%; p>0,05), porém, em ambas as avaliações, as participantes podem ser classificadas como ativas (escore $\geq 2,08$ pontos). Apesar do decréscimo nesse domínio não ser significativo, o NAF_E é o de maior impacto fisiológico na demanda energética, sendo assim, o principal contribuinte para o escore do nível de atividade física total, como indicado em outros estudos ${ }^{12,13,14}$.

Apesar de considerar as participantes deste estudo como ativas, outros estudos demonstram que o nível de atividade física de idosas praticantes de exercícios físicos pode ser muito superior ao encontrado nesta amostra. Miyasike da Silva et al $(2000)^{12}$, realizou um estudo com indivíduos de 50 à 75 anos, utilizando o mesmo instrumento desta pesquisa, encontrando uma média do NAF_T de 3,19 pontos para um grupo de sedentários; porém de 7,82 e 8,53 pontos em outros dois grupos de indivíduos ativos. Ademais, Gobbi et al $2007^{13}$ conduziu uma pesquisa em Rio Claro, São Paulo com 60 mulheres ativas (50 e 70 anos), utilizando também o mesmo instrumento. A amostra foi dividida em dois grupos: participantes de um programa de atividade física supervisionada (GS) e não supervisionada (GNS), as quais reportaram médias de NAF_T de 12,73 pontos para o GS, e de 7,48 pontos para GNS (diferença significativa entre grupos, $\mathrm{p}<0,05$ ). Alencar et al $(2010)^{14}$ reportaram um nível de atividade física insuficiente em idosas residentes na zona rural de Crato-CE devido ao fato de nenhuma idosa relatar a prática de exercícios físicos. Desta forma, o principal responsável pelo NAF_T foi o domínio doméstico seguido pelo recreativo. Os autores justificam o fato pela "inexistência de programas que estimulem a prática de atividades físicas na população em geral, 
principalmente em idosos”. Esses resultados sugerem que a prática somente das atividades relacionadas aos domínios doméstico ou de recreação não garantem um elevado nível de atividade física ${ }^{6,13,14}$.

Os resultados apresentados indicam que para se obter um elevado nível de atividade física é necessário que idosos participem de um programa regular de exercícios físicos e não apenas sejam considerados como ativos. Ademais, a participação em um programa de exercícios físicos aumenta as chances do idoso tornar-se mais eficiente para a execução de outras atividades, bem como da manutenção das capacidades físicas e funcionais, essenciais para um envelhecimento saudável ${ }^{6,28}$. Sendo assim, as alterações no NAF_E, necessitam de uma análise cautelosa pois a diminuição de exercícios físicos pode impactar com diferentes magnitudes aspectos sociais, de saúde e, consequentemente, da qualidade de vida de idosos ${ }^{10}$.

Os achados no presente estudo demonstram uma ampla variabilidade no NAF_E, indicando que 32,05\% das idosas modificaram sua prática de exercícios de uma intensidade leve para moderada, da primeira para a segunda avaliação; $29,48 \%$ mantiveram atividades em intensidade leve; enquanto que $20,51 \%$ daquelas que praticavam exercícios físicos de intensidade leve abandonaram a prática. Cerca de $8,97 \%$ de idosas na primeira avaliação relataram não praticar qualquer exercício físico, aumentando para $24,36 \%$ na segunda avaliação. Além disso, não houve relatos de prática esportiva de intensidade vigorosa na primeira avaliação, mas três idosas $(3,84 \%)$ reportaram praticar atividades físicas de alta intensidade na segunda avaliação. Enquanto algumas idosas aumentaram a intensidade dos exercícios realizados, elevando o escore do NAF_E, a maioria abandonou esta prática, diminuindo o escore do NAF_E. Este fato justifica parcialmente o motivo pelo qual as alterações neste domínio não foram significativas. O principal exercício reportado foi a caminhada, o qual foi observado recentemente em idosos residentes em Florianópolis-SC ${ }^{29}$ e também em Ermelino Matarazzo-SP ${ }^{30}$, seguido pela ginástica, hidroginástica, musculação e natação.

Os dados longitudinais da segunda avaliação contradizem os resultados publicados anteriormente da primeira avaliação ${ }^{9}$, na qual foi encontrada uma tendência de transição da intensidade das atividades realizadas pelas idosas. Com o avanço da idade, principalmente a partir da faixa etária de 65-69 anos, diminuiu a realização de atividades esportivas de intensidades moderadas-vigorosas (NAF_E), e aumentou a realização de atividades recreativas de baixa intensidade (NAF_R) 9 . Certamente, as atividades ou exercícios físicos que exigem elevada demanda energética seriam as mais recomendadas para idosos aparentemente saudáveis visando a melhoria da saúde física. No entanto, ao se tratar do indivíduo idoso, é essencial que também se priorize a saúde emocional e psicossocial que podem apresentar efeitos benéficos por meio de qualquer atividade física que proporcione o convívio e exposição social, bem como o desenvolvimento cognitivo.

Após a busca em diversas bases de dados científicos (PUBMED, SCIELO, LILACS e também no portal PERIÓDICOS CAPES), não foi encontrado nenhum estudo Brasileiro que investigou as alterações longitudinais em domínios do NAF, especificamente em mulheres idosas. Este fato também foi exposto por outros pesquisadores, os quais reportaram a necessidade de estudos que verifiquem não apenas a magnitude dessas alterações ao longo do tempo, mas também que analisem a influência de fatores sociodemográficos, ambientais, de saúde física, mental ou funcional no NAF ${ }^{27,29,30,31}$. Recentemente foi demonstrado que as mulheres de baixo nível socioeconômico ou educacional apresentam a maior prevalência de inatividade física no tempo livre ${ }^{10,26}$; que homens e mulheres idosos mais 
ativos no tempo livre são aqueles que percebem a existência de pista de caminhada no bairro, ciclovias, que recebem apoio de amigos ou vizinhos, ou são influenciados por fatores climáticos ${ }^{29}$; todavia, especificamente idosas ativas no lazer são aquelas que residem próximas a igrejas ou templos religiosos, academias e praças ${ }^{30}$. Sendo assim, futuras pesquisas podem preencher tais lacunas, realizando uma análise longitudinal em domínios específicos do NAF e a influência desses fatores. Este estudo coletou informações sócio demográficas, de saúde física e funcional das idosas, mas uma análise estatística mais robusta foi impossibilitada devido ao pequeno número de participantes na segunda avaliação. Outra limitação deste estudo foi o uso de informações auto relatadas, mesmo assim, os resultados apresentados evidenciam que o declínio nas atividades comumente realizadas por idosas ocorrem nos três domínios do NAF. Essas evidências alertam que intervenções necessitam ser realizadas a fim de conscientizar idosas sobre os benefícios da manutenção de um estilo de vida ativo.

\section{CONCLUSÃO}

Este estudo indica que com o avanço da idade ocorre um declínio no nível de atividade física de idosas, sendo de maior magnitude nas atividades de lazer/recreação e menor magnitude nas atividades relacionadas ao cuidado domiciliar. Apesar das participantes desta amostra serem classificadas como ativas, os resultados são inferiores aos reportados em outras cidades brasileiras. Além disso, foi constatado que quase metade das idosas são sedentárias (não praticam nenhum tipo de exercícios físicos). Esses dados demonstram a necessidade de maximizar políticas públicas para o cuidado à saúde do idoso a fim de promover atividades nos diferentes domínios do NAF, e motivar idosos a manterem uma vida ativa e independente, principalmente participando regularmente em um programa de exercícios físicos, o qual acarretará em consequências benéficas na qualidade e nos anos adicionais de vida desta população.

\section{Agradecimentos}

Ao Conselho Nacional de Desenvolvimento Científico e Tecnológico - CNPQ pelo apoio financeiro a este projeto (479491/2010-0); edital MCT/CNPq No 014/2010 - Universal.

\section{Contribuição dos Autores}

Todos os autores, Gisele Antunes do Livramento, Pallomma Patrícia Andressa Nart Fagundes, Gabriele Regiane Winter, Vanessa Porto Bernardes e Maressa Krause, participaram ativamente na elaboração do manuscrito no sentido de redigir o texto. Maressa P Krause coordenou a coleta de dados, orientou a proposta do artigo, realizou as análises estatísticas e a revisão final do mesmo.

\section{REFERÊNCIAS}

1. Silva TAA, Frizoli Junior A, Pinheiro MM, Szejnfeld VL. Sarcopenia Associada ao envelhecimento: Aspectos etiológicos e opções terapêuticas. Rev Bras Reumatol 2006; 46(6): 391-397.

2. Harold W, Kohl III. Physical activity and cardiovascular disease: evidence for a dose response. Med Sci Sports Exerc 2001;33(6):472-483.

3. Matsudo SM, Matsudo VKR, Neto TLB. Atividade física e envelhecimento: Aspectos epidemiológicos. Rev Bras Med Esporte 2001;7(1):02-13. 
4. Matsudo SM, Matsudo VKR, Araujo T, et al. Nível de atividade física da população do Estado de São Paulo: Análise de acordo com o gênero, idade, nível socioeconômico, distribuição geográfica de conhecimento. Rev Bras Ciênc Mov 2002;10(4):41-50.

5. Eyler AA. The epidemiology of walking for physical activity in the United States. Med Sci Sports Exerc 2003;35:1529-1536.

6. Brasil. Ministério da Saúde. Secretaria de Vigilância em Saúde. Secretaria de Gestão Estratégica e Participativa. Vigitel Brasil 2010: vigilância de fatores de risco e proteção para doenças crônicas por inquérito telefônico / Ministério da Saúde, Secretaria de Vigilância em Saúde, Secretaria de Gestão Estratégica e Participativa. - Brasília: Ministério da Saúde, 2011.

7. Alencar NA, Souza Junior JV, Aragão JCB, Ferreira MA, Dantas E. Níveis de atividade física, autonomia funcional e qualidade de vida em idosas ativas e sedentárias. Fisioter Mov 2010; 23(3):473-481.

8. Secretaria de Políticas de Saúde. Programa Nacional de Promoção da Atividade Física "Agita Brasil": atividade física e sua contribuição para a qualidade de vida. Rev Saúde Públ 2002; 36(2):254-256.

9. Krause MP, Buzzachera CF, Hallage T, Pulner SB, Silva SG. Influência do nível de atividade física sobre a aptidão cardiorrespiratória em mulheres idosas. Rev Bras Med Esporte 2007;13(2):97-102.

10. Hallal PC, Dumoith SC, Bastos JP, et al. Evolução da pesquisa epidemiológica em atividade física no Brasil: revisão sistemática. Rev Saúde Públ 2007; 41(3):453-460.

11. Matsudo SM, Marin RV, Ferreira MT, Araujo TL, Matsudo V. Estudo longitudinal - tracking de 4 anos - da aptidão física de mulheres da maioridade fisicamente ativas. Rev. Bras. Ciênc Mov. 2004; 12(3): 47-52.

12. Silva Junior JP, Silva LJ, Ferrari G, et al. Estabilidade das variáveis de aptidão física e capacidade funcional de mulheres fisicamente ativas de 50 à 89 anos. Rev Bras Cineantropom Desempenho Hum 2011; 13(1):08-14.

13. Miyasike da Silva V, Gonçalves CT, Silva JJ, Gobbi LTB. Mobilidade de idosos em ambiente doméstico: efeitos de um programa de treinamento específico. Rev Bras Ativ Saúde 2003; 8(1): 05-19.

14. Gobbi S, Menezes E, Tanaka K. Programas supervisionados de atividades físicas estão associados a maior nível de atividade física comparados com não supervisionados. Rev Bras Ativ Fís Saúde 2007; 12(1):03-07.

15. Alencar NA, Aragão JCB, Ferreira MA, Dantas EHM. Níveis de atividade física em idosas. Estud Interdiscipl Envelhec 2010;15(1):87-97.

16. Duca GF, Rombaldi AJ, Knuth AG, et al. Associação entre nível econômico e inatividade física em diferentes domínios. Rev Bras Ativ Fís Saude 2009;14(2):123-131.

17. Associação Nacional das Empresas de Pesquisa - ANEP. Critério de classificação Econômica Brasil 2003. http:// www.anep.gov.br. Acesso em 15 de junho de 2010.

18. Lohman TG, Roche AF, Martorell R. Anthropometric Standardization Reference Manual Abridged Edition. Champaign, IL: Human Kinetics.1988.

19. Voorrips LE, RavelliACJ, Dongelmans PCA, Deurenber P, Van Staveren WA. A physical activity questionnaire for the elderly. Med Sci Sports Exerc 1991;23:974-970.

20. Alencar NA, Bezerra JPC, Dantas EHM. Avaliação dos níveis de atividade física, autonomia funcional e qualidade de vida de idosas integrantes do programa de saúde da família. Fit Perform J 2009;8(5):315-321.

21. Mazo GZ, Mota J, Benedetti TB, Barros MVG de. Validade concorrente e reprodutibilidade: Teste-reteste do questionário baecke modificado para idosos. Rev Bras Ativ Fís e Saúde 2001; 6(1):05-11.

22. Oliveira MM, Maia JA. Avaliação da actividade física em contextos epidemiolóigicos. Uma revisão da validade e fiabilidade do acelerometro Tritrac-R3D, do pedometro Yamax DigiWalker e do questionário de Baecke. Rev Port Ciênc Desporto 2001; 1(3):73-88.

23. Camarano AA. Envelhecimento da população brasileira: uma contribuição demográfica. Instituto de Pesquisa Econômica Aplicada (IPEA). Texto para discussão no 858; janeiro, 2002.

24. Paiva ACS, Hernandez SS, Quadros Jr AC, et al. Dança e envelhecimento: Uma parceira em movimento! Rev Bras Ativ Fís Saúde 2010; 15(1):70-72.

25. Oliveira LC, Pivoto EA, Vianna PCP. Análise dos resultados de qualidade de vida em idosos praticantes de dança sênior através do SF-36. Acta Fisiátrica 2009; 16(3):101-104. 
26. Galisteu KJ, Facundim SD, Ribeiro RCHM, Soler ZASG. Qualidade de vida de idosos de um grupo de convivência com a mensuração da escala de Flanagan. Arq Ciênc Saúde 2006;13(4):209-214.

27. Zaitune MPA, Barros MBA, César CLG, et al. Fatores associados à prática de atividade global e de lazer em idosos: Inquérito de Saúde no Estado de São Paulo (ISA-SP), Brasil. Cad Saúde Pública 2010;26(8):1606-1618.

28. Duca GDF, Thume E, Hallal PC. Prevalência e fatores associados ao cuidado domiciliar a idosos. Rev Saúde Públ 2011; 45(1):113-120.

29. Giehl MWC, Schneider IJC, Corseuil HX, Benedetti TRB, d'Orsi E. Physical activity and environment perception among older adults: a population study in Florianópolis, Brazil. Rec Saúde Pública 2012;46(3):1-9.

30. Salvador EP, Florindo AA, Reis RS, Costa EF. Percepção do ambiente e prática de atividade física no lazer entre idosos. Rev Saúde Publica 2009;43(6):972-980.

31. Tribess S, Virtuoso-Júnior JS, Petroski EL. Fatores associados à inatividade física em mulheres idosas em comunidades de baixa renda. Rev Salud Pública 2009;11(1):39-49.

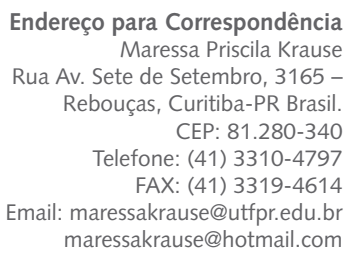

